\title{
Compensation of active and reactive power in PV- WIND battery system by using ANN technique
}

\author{
A. Ashwini ${ }^{1, *}, V$. Usha Rani $^{2}, J$. Sridevi $^{3}$ \\ ${ }^{1}$ PG student, EEE Department, GRIET, Hyderabad, Telangana \\ ${ }^{2}$ Assistant Professor, EEE Department, GRIET, Hyderabad,Telangana \\ ${ }^{3}$ Professor, EEE Department, GRIET, Hyderabad, Telangana
}

\begin{abstract}
This paper proposes the improvement of PV-WE system's energy usage and the battery energy storage system (BESS). PV, BESS. Surveillance, tension management, frequency control, energy distribution, power quality and artificial intelligence techniques (AI). The aim is to increase the power quality of the grid-connected PV-BESS system. In order to increase energy quality, technology is continually explored and evaluated. The PVBESS system is built for the microgrid, which offers benefits including continuous supply, efficient load content and effective electricity utilisation. The flow of energy from source to source is controlled by ANN. The development of the MPPT algorithm for validation of the proposed approach is using an artificial neural network (ANN) technique. An extensive research and data finding demonstrates that output is accuracy with the ANN-based MPPT output. This study therefore proposes a new way for assessing the performance at a specific site of the microgrid. The power translation systems are therefore handled in an active and reactive manner taking into consideration their circumstances and limitations.
\end{abstract}

\section{Introduction}

The power balancing and load sharing are hierarchical control systems for voltage/frequency control, such as primary monitoring, secondary control and tertiary control[1], that are employed as a microgrid control. The decentralised drop-based control system also gives without communication an essential alternate control[2]. However, the following shortcomings are shown in various control systems:

\subsection{System Coordinates}

Generally, models were developed in the $d-q$ synchronous frame of reference. However, this strategy presents some inconveniences. First of all, this technique takes the form of properly balanced three phase systems for loads, grids and models. However, a load normally involves several single-phase loads spanning three phases, and so a perfect load balancing, especially in a microgrid, cannot be achieved. A load does not necessarily represent a linear load and can be distorted or harmonic very easily. Models are therefore employed in this research in abc co-ordinates.

\subsection{PLL (Phase locked loop)}

In practise it needs to be monitored, and PLLs have been employed for this. This is a realistic technique. PLLs have their reactivity and characteristics, and both simulation models and control techniques have these characteristics. For the monitoring of the several models, the angles d-q to a-b-c and a-b-c to d-q are needed (e.g. the 3-phase inverter model). This work aims at the improving the dynamic performance of the available photovoltaic system.

\subsection{Controller}

A control approach that selectively limits the output of the control, taking into account also model line impedances, to achieve the control target.

In tandem with the RESs, the converters are utilised for transforming power into the grid. In the reference study[12], RES is connected to a bidirectional transformer and a hybrid configuration layout that includes PV and WE. Different characteristics like average wind speed, radiation, and temperature need to be taken into account when calculating PV and WE systems as weather-sensitive RESs. In several nations like India, China, Iceland, Sweden and the USA, there are a wide range of RES scenarios[2]. To be added in WE, on-shore and offshore output capacity is different[13]. Accordingly, flexible mathematical modelling and optimization methodologies must be designed to design seasonable WE and PV systems according to the many aspects affecting PV and WE systems. It is difficult to design the non-linear features of pv. Various optimization methods like as the optimisation of particulate swarm are therefore employed for estimating PV system parameters and improving the power production of PV and WE [14]. Ultimately, these strategies will aid configure the modelling of RESs. However, due to voltage drops on the line[4], the distributed generation (GD) units observed incorrect reactive power sharing. In order to overcome these restrictions[1],[3],[5]-[11] A secondary central control that requires dual-way communication 
between the central control and each DG unit. However, there are no constraints on zero error voltage and frequency rules on the secondary control system[12]. Moreover, although for the correct analysis of practical microgrid systems of low voltage, the line impedances should be considered for the system model, the provided research neglects the effect of these parameters[13],[14]. Motivated by the above restrictions, these papers introduce a new technique of supervising the power control of the grid-off microgrid at a given location, i.e. PCC Micro grid-off. The approach used recognises that, given the availability of power sources and their contribution to load consumption, each DG unit has somewhat differing instantaneous active/reactive power output. Consequently, the key control targets will be attained with varied levels of active and reactive power accessibility. In addition to ensuring that every DG unit operates within a steady region of operation, operational limits are in place. The key topic of this study is as follows in the simulation environment:

- PV-BESS system power augmentation.

- Removal of grid voltages and currents of THD.

- Good voltage control under different load circumstances.

- To have enough power to meet the demand for load in the worst circumstance in which RES cannot be generated by electricity.

- Use EVs as a surplus power supply and as an electricity supplier while requiring large grid loads. In varied weather situations, the MPPT algorithm greatly contributes to the highest power generation. For PV and BESS systems in the MATLAB environment, the PI and ANN controllers are created. Finally, both controllers compare the simulation results with the best.

\section{Methodology}

The effort focuses on improving the power and grid stability of BESS and non-linear loads for RESs. There will be a discussion of solar PV and BESS mathematical modelling systems in this section. This section also contains the MPPT algorithms and design procedures.

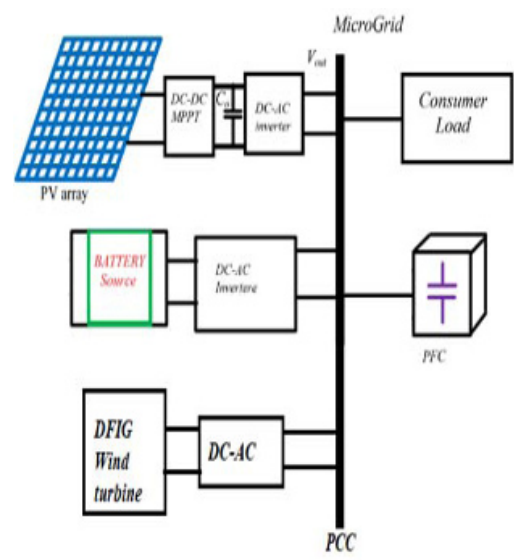

Fig. 1. Branches of the PV-wind system.

\section{Modelling of solar PV system}

In order to extract maximum power out of a PV panel[4]-[6], several of the researchers investigated MPPT models and algorithms in the literature. Figure 2 illustrates the circuit programme of an easy PV system. The authors have defined in detail in [4][6] the mathematical equation of the panel's I-V characteristics. The panel's current output is IL, the diode current is Idiode, the PV panel current is IS, the heat voltage is VT, $\mathrm{c}$ is diode constant and the Rseries and Rparallel are shown in series and in parallel branches, and isate is reverse saturation.

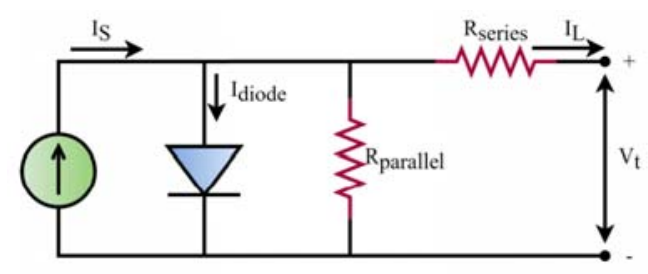

Fig.2. Single solar PV panel circuit diagram

$$
I_{L}=I_{S}-\left[\exp \left(\frac{V_{t}+R_{\text {series }} I_{L}}{c V_{T}}\right)-1\right]-\frac{V_{t}+R_{\text {series }} I_{L}}{R_{\text {parallel }}}
$$

MPPT algorithm panel block scheme is shown in Fig. 3. The bad impacts of the normal MPPT algorithm are removed by means of the MPPT algorithm based on ANN [4][7], including poor unit shading performance and the local maximum power point for partial radiation selection. ANN is the most used MPPT algorithm technology in the recent epoch. This study therefore develops ANN-based MPPT.

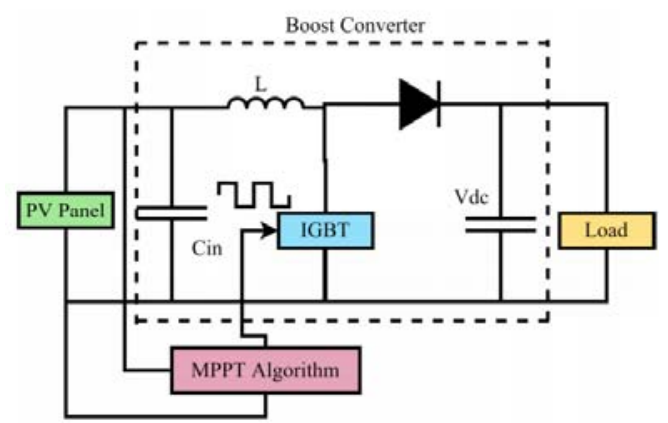

Fig.3. MPPT Converter and boost converter for extracting the panel's maximum power output.

\section{Soc estimation model}

Precision dynamic battery modelling is necessary so that numerous challenges confronting the old models can be overcome. The ampere-hour counting approach fits well for calculating the status of the charge, as described in Reference [21]. The time to load or download and the current expression can be set as follows this model 


$$
S o C=S o C_{0}+\int_{t_{0}}^{t}\left(\frac{I_{\text {battery }}}{C_{\text {battery }}}\right) d \tau
$$

Where SoC $0=$ the battery's original SOC point, $\mathrm{I}_{\text {battery }}=$ the current of the battery, and $\mathrm{C}_{\text {battery }}=$ battery capacity. The batteries can be charged and discharged via SoC equation as indicated in Equation.

$$
S o C=S o C\left[1-\frac{\sigma}{24}\left(t-t_{0}\right)\right]+\int_{t_{0}}^{t}\left(\frac{I_{\text {batter }} \eta_{\text {battery }}}{C_{\text {battery }}}\right) d \tau
$$

Where $\mu=$ rate of self-de loading; battery = efficiency of battery charging or discharge.

The capacity of the battery depends on the temperature and is:

$$
C_{b a t t e r y}=C_{\text {battery }}^{\prime}\left(1+\delta_{c}\left[T_{\text {battery }}-298.15\right]\right)
$$

Where the battery = capacity of the battery is when the battery temperature is $\mathrm{T}$ battery and $\mathrm{C} 0=$ nominal capacity of the battery.

\section{Phase locked loop (PLL)}

In particular in unbalanced or distorted cases, PLL's PLL Single Order General Integrator (SOGI) is meant to deliver enhanced performance of PLL. G. Power factor compensation (PFC) The three-phase reactive power compensator is a model of the delta-connected condensers[23]. This block has an actual input and the voltage is created on the condenser terminals. It can be used for blocks generating a true output (voltagecontrolled current sources). The three-phase reactive power compensator control determines the required setting based on the measured capacity of the reactive power compensator block. Near the unit the power factor should be changed.

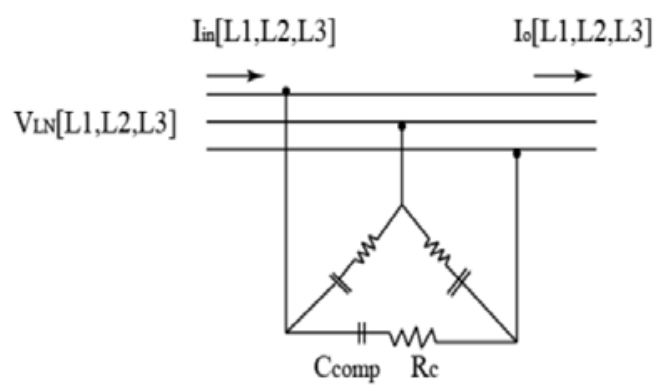

Fig. 4. Schematic compensating factor power (voltage input).

\section{Supervisory power quality control scheme}

This section contains a new monitoring system for the quality control of power not requiring the installation of additional compensatory devices and adaptable to various configuration microgrids. The controller manages the voltage/frequency of each DG power conversion system by means of modifications to the operating limits for active/reactive power. Due to availability of the source of energy and the changes in demand, each DG is able to accomplish a variation in a realistic micro-grid instantaneous power output. Consequently, the active/reactive power generating system likewise has varying availabilities.

\section{Artificial neural network control scheme}

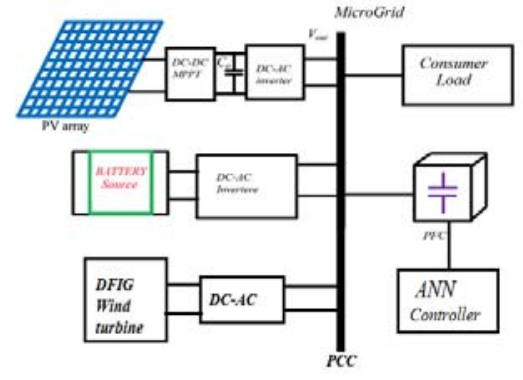

Fig.5. Artificial neural network control scheme

Here in this paper ANN controller controls the Active and Reactive power by operating the grid inverter with the Power factor correction controlled by PLL and the Power quality control. Artificial neural networks (ANN) are generally described as the interconnected "neurons" system for the exchange of signals. These links are numerical, input-adaptable, and learning able, based on experience. ANN is one of the areas of AI technology. This concept provides an alternative approach to the power system application. The network has neurons, neurons and 2 hidden layers, which flow from the network through the output layer. As an input-output model, the network is basic and its weights are as one parameter. The multi-layer $\mathrm{NN}$ architecture is shown in Figure 6.

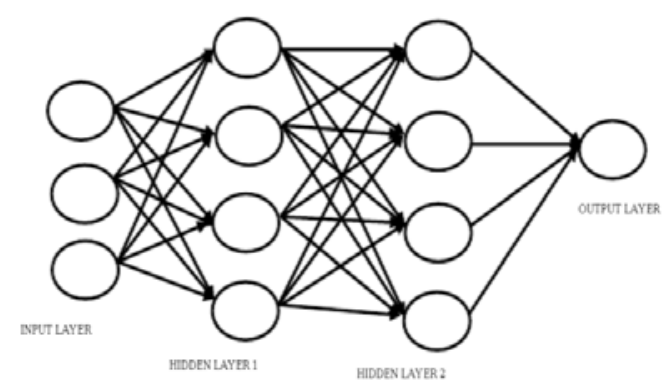

Fig.6. The multi-layer architecture of the neural network.

There are two paths in the training cycle: first is forward spreading and second is error reverse spreading. The first way sends the inputs through the neural network structure to their output.

To reduce the output error, the weights are adjusted. The Marquardt Levenberg training algorithm is used to train neural network monitoring and is effective, it is effective and efficient easy to build and takes no time[14]-[18]. The layer comprises of 1 layer of input, 2 layer and 20 neurons and one layer of output through network training. The input layer has a tan-sigmoid 
feature and Pos-Linear is activated with the output layer. The neural network is constructed by applying the BP algorithm for the set neurons of each layer. 323 iterations and errors are made during every training session.

\section{Simulink results}

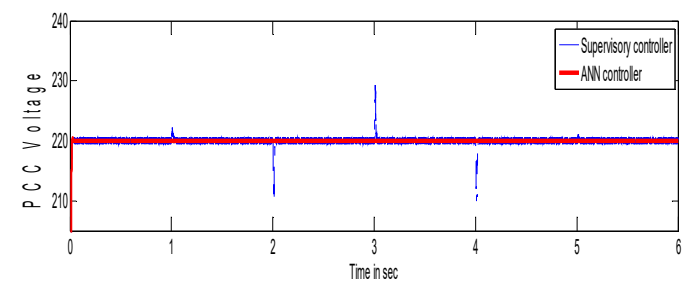

Fig. 7. Comparison of ANN and supervisory controller PCC Voltage variations

In the above Fig7. Supervisory controller at PCC has many disturbances when compared to $\mathrm{ANN}$, at time periods $t=1$ to 6 at every operation there is a disturbance in the PCC voltage in the Supervisory controller, whereas ANN doesn't have any disturbances.

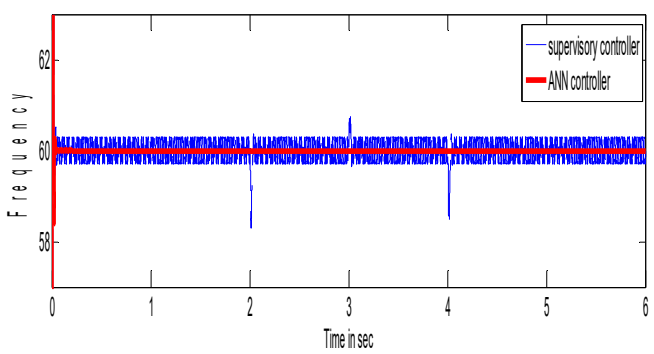

Fig. 8. Comparision of ANN and supervisory controller frequency deviations.

Fig8. Shows the deviations in the frequency with the supervisory controller and ANN controller whereas the deviations accours due to load variations and the source vartions in the Micro grid.

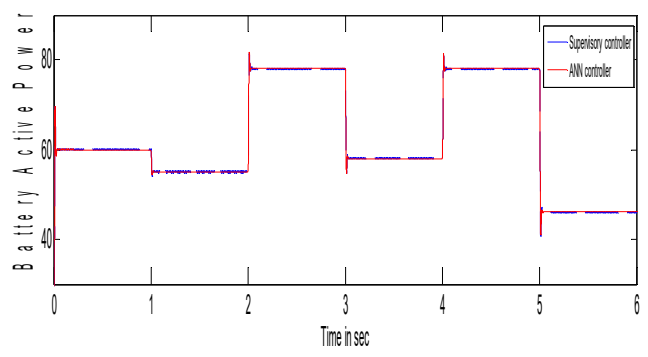

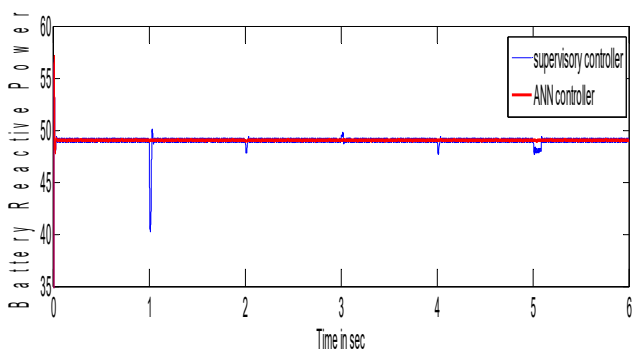

Fig. 9. Variation of Battery Active and Reactive powers in ANN and Supervisory controller.

Fig9 Describes the BESS Active and reactive power compensations of the Micro grid by the SPF
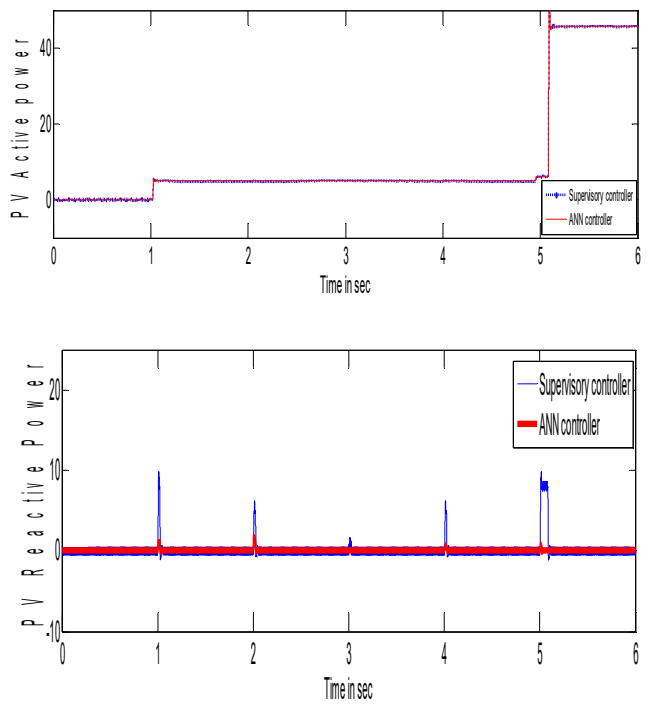

Fig. 10. PV active and reactive power fluctuations with ANN and Supervisory controller

Fig10. Shows the PV active and reactive power after the MPPT technique of the PV system. By supervisory controller the MPPT ooperation and the Active and reactive power was poorly developed in the PV system. By the proposed controller there is an improvement in the PV system.

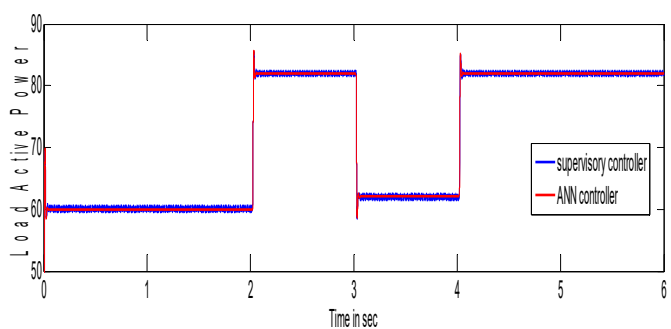




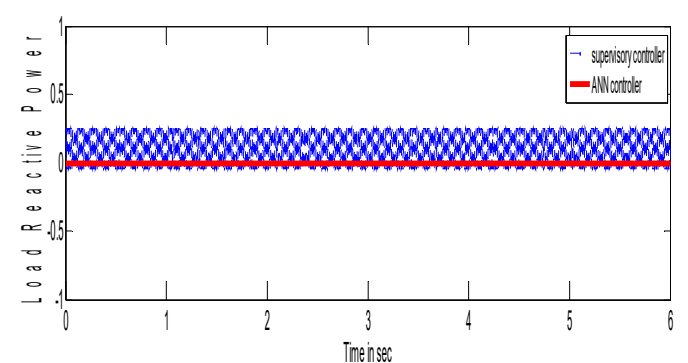

Fig.11. ANN and Supervisory controller active and reactive power fluctuations of load.

In the Fig 11, active and reactive power fluctuations of load side are shown here the fluctuations with Supervisory controller are in high when compared to the ANN. ANN controls the fluctuations better than the Supervisory controller.

\section{Conclusion}

A technique for the improvement of power of PV-BESS with ANN controls has been proposed by the authors. The MPPT algorithms based on ANN are designed for the extraction of maximum power by PV and BESS. The shunt active filter is accomplished in non-linear terms with the use of ordinary PI controllers and artificial intelligence networks ANN. The proposed ANN control technique achieved the improvement of harmonic supplies currents and reactive energy consumption via non-linear load. The enhanced efficiency of the calculation approach to artificial intelligence shows that a range of challenges of power quality can be used. Active and reactive power of the Micro grid is controlled by the ANN based shunt active filters. The THD at the load and source voltages and currents are controlled by the proposed controller, which complies with THD's threshold limits according to the IEEE-519 standard (1992). Finally, the ANN method gives better results compared to the PI controller.

\section{References}

1. R. M. Elavarasan, Eur. J. Sustain. Develop. Res., 3, (2019)

2. R. M. Elavarasan, J. Sol. Energy Eng., 142, 111, (2020)

3. R. Madurai Elavarasan, S. Afridhis, R. R. Vijayaraghavan, U. Subramaniam, and $\mathrm{M}$. Nurunnabi, Energy Rep., 6, 1838-1864, (2020)

4. R. Madurai Elavarasan, L. Selvamanohar, K. Raju, R. R. Vijayaraghavan, R. Subburaj, M. Nurunnabi, I. A. Khan, S. Afridhis, A. Hariharan, R. Pugazhendhi, U. Subramaniam, and N. Das, Sustainability, 12, 6596, (2020)

5. G. M. Shafiullah, M. T. Arif, and A. M. T. Oo, Sustain Energy Technol. Assessments, 41, 631640, (2020)

6. S. A. Mohamed, Int. Trans. Electr vol 10, (2018)

7. L. Ashok Kumar and V. Indragandhi, Int. J. Energy Syst., 30, 12196, (2020)
8. S. Paramanik, K. Sarker, D. Chatterjee, and S. K. Goswami, in Proc. Devices for Integr. Circuit (DevIC), 23, 356-360, (2019)

9. J. Schiffer, T. Seel, J. Raisch, and T. Sezi, IEEE Transactions on Control Systems Technology, 24, 96-109, (2016)

10. Q. Shafiee, J. M. Guerrero, and J. C. Vasquez, IEEE Transactions on Power Electronics, 29, 1018-1031, (2014)

11. L.-Y. Lu and C.-C. Chu, IEEE Transactions on Power Systems, 30, 2243-2256, (2015)

12. J. W. Simpson-Porco, F. D"orfler, and F. Bullo, Automatica, 49, 2603-2611, (2013)

13. X. Wu, C. Shen, and R. Iravani, IEEE Transactions on Smart Grid, 17 (2016)

14. Y. W. Li and C.-N. Kao, IEEE Transactions on Power Electronics, 24, 2977-2988, (2009)

15. Maniktala, Sanjaya: Switching Power Suppliers A-Z, 2nd Ed., Newnes Press, 15 (2012)

16. R. W. Erikson, D. Maksimovic: Fundermentals of Power Electronics, 2nd Ed., Kluwer Academic, 40, (2001)

17. H. S. Ko, T. Niimura, J. Jatskevich, H. C. Kim, and K. Y. Lee, in IEEE Power Engineering Society Meeting, Denver, Colorado, 26, 17221728, (2004)

18. G. Perez, I. Gandiaga, M. Garmendia, J. F. Reynaud and U. Viscarret, International Battery, Hybrid and Fuel Cell Electric Vehicle Symposium, 43, 1-9, (2013)

19. N. Femia, G. Petrone, G. Spagnuolo, M. Vitelli, CRC Press, 16, (2013)

20. R. Teodorescu, M. Liserre, P. Rodriguez: Wiley-IEEE Press, 8, (2011)

21. Nutakki, M., Palakaluri, S.V.D., Vijaya Santhi, R. ;International Journal of Innovative Technology and Exploring Engineering, 11,2325-2329, (2019)

22. Sai, V.A., Srikanth, B., Devi, P.S., Rajamahanthi, V.; International Conference on Sustainable Energy and Future Electric Transportation, SeFet, 6, (2021)

23. Jayahari, L., Hussaini, S.M., Varmaa, D., Advances in Materials and Processing Technologies, 2 ,285-295, (2020)

24. Srividya Devi.P, R Vijaya Santhi International Journal of Electrical and Computer Engineering, 9 ,861, (2019)

25. DSNMRAO, Ch. Pushpa Latha, N. Bharath kumar and PM Venkatesh, Journal Européen des Systèmes Automatisés (JESA), 52, 535540, (2019)

26. Adapa, R.R., Nawaz, S.S., Padhan, D.G. Proceedings - 2020 IEEE India Council International Subsections Conference, INDISCON 7, 9344573, (2020)

27. Reddy, K.L.P.K., Padhan, D.G. E3S Web of Conferences, 84, (2020) 